\title{
Direct Drive for Low Power Hall Thrusters
}

\author{
John W. Dankanich* \\ Gray Research Inc., Huntsville, AL, 35824
}

\begin{abstract}
Due to recent studies, NASA has initiated the development of a low power Hall thruster for discovery class missions. The potential advantages of a low power Hall thruster is primarily due to its high efficiency operation at low power and its lower complexity compared to ion engines. Direct drive is another method of reducing the complexity of a Hall thruster system while improving its efficiency. The technical challenges associated with this technology are reported. Additionally, the benefits of this technology are discussed based on parametric studies and mission analysis.
\end{abstract}

\section{Introduction}

$\mathrm{T}$ he NASA Refocus studies ${ }^{3,5}$ were conducted by the In-Space Propulsion Technology Program to help focus investments into technologies that can enable or benefit near and mid-term NASA space science missions by significantly reducing cost, mass, and trip times. One result of the studies has shown that the low power High Voltage Hall Accelerator (HiVHAC) system has significant mission capture for Discovery Class missions through its ability to operate efficiently at very low power levels and its decreased complexity over the state of the art NSTAR ion thruster. Hall thrusters in general are less costly than ion engines to manufacture due to their simplicity in design, lack of accelerator grids, and ability to function without an independent digital control interface unit (DCIU). Typically reduction in mass and complexity results in reduced cost for spacecraft. Direct drive has often been considered a significant cost saver for Hall thruster technology due to its reduction is mass, complexity, and thermal burden to the spacecraft. Both the direct drive mass savings and thruster system performance increase as the available thruster power increases. This paper shows the results of a study to determine if the benefits remain significant with the implementation of direct drive on a low power system where a higher specific impulse is desired.

\section{Direct Drive Applicability}

The concept of a direct drive electric propulsion system (EPS) has been in literature for decades. Direct drive is removing the majority, virtually eliminating, the power processing unit (PPU) from an EPS. The advantages of a direct system include increased efficiency and decreased system mass. Also, the elimination of flight hardware elements will typically reduce costs of flight qualification and system integration while improving system reliability. Recent improvements in solar array technology allow for high voltage, greater than $400 \mathrm{~V}$, solar arrays that are well suited for direct drive Hall thruster applications.

PPU efficiencies are typically on the order of $90 \%$, but the $10 \%$ losses are not insignificant. The heat lost due to PPU inefficiencies must be dissipated by the thermal control system with typical alphas of $15-30 \mathrm{~kg} / \mathrm{kW}$. The increased efficiency also allows for a smaller solar array to perform the same mission with a reduced mass and cost of $\sim 20 \mathrm{~kg} / \mathrm{kW}$ and $\$ 2 \mathrm{M} / \mathrm{kW}$. For high power solar electric propulsion (SEP), there are large mass and cost savings. For theoretical calculations, this study assumes a standard PPU has a constant efficiency of $92 \%$ and a direct drive PPU has an efficiency of $99 \%$ at all power levels.

Another benefit of direct drive is the simplification of the EPS and spacecraft integration. Complexity is difficult to quantify, however; the PPU provides power to thruster while meeting interface and compatibility requirements with the spacecraft and additional subsystems. A major burden on the spacecraft is the thermal loading from the PPU that must be dissipated. Direct drive significantly reduces this waste energy reducing spacecraft integration requirements.

One method the HiVHAC system can achieve its performance characteristics is by throttling its voltages along with its power input as the spacecraft moves farther from the Sun and experiences its inverse square power loss. HiVHAC is therefore a variable specific impulse Hall thruster. When more power is available to the thruster, the propulsion system optimizes towards a higher voltage, approximately 800 volts at $2.8 \mathrm{~kW}$; and therefore a higher $I_{\mathrm{sp}}$ than possible with a 400 volt direct drive system. On the low power end of the spectrum, the optimized system

\footnotetext{
* Aerospace Engineer, NASA In-Space Propulsion Technology Project Office, NASA MSFC M/S NP-40.
} 
tends to desire a lower voltage, approximately 300 volts at 300 Watts, to maintain desired thrust levels. This effect is illustrated in figure 1, with HiVHAC representing the optimal operating condition.

With a direct drive system the voltage cannot be controlled. In fact, as the spacecraft moves farther from the sun, the voltage generated by the solar array may increase slightly due to low temperature low intensity (LTLI) effects. The Deep Space-1 ion propulsion demonstrator observed a voltage increase of approximately $23 \%$. State of the art solar cells have minimal LTLI effects, however; even slight increases in voltage when decreased voltage is desired will have negative effects on performance. In actuality, because the desired voltage is higher than the design point, as long as sufficient currents can be achieved, the higher specific impulse may yield better performance. The LTLI effects may play a more negative role if the system was originally optimized for $I_{s p}$ and not limited to voltage arcing constraints.

In the absence of a PPU, the specific impulse profile will be relatively flat during the mission and the discharge current will be proportional to the flow rate into the anode chamber of the thruster; which must be thermally controlled by a feed system orifice. The result is a thruster that will produce a higher $I_{s p}$ exhaust where a higher thrust is preferred at the very low power levels, and a higher thrust will be produced at the higher power levels when a higher $I_{s p}$ is desired. Intuitively, one would think there is a point where the savings in mass and increased power efficiencies of a direct drive system would be overshadowed by the decrease in performance by running the thruster in a non-optimized configuration for the majority of the mission. Because this study looks at missions where the desired $I_{s p}$ is above those achievable at $400 \mathrm{~V}$; and the very low power levels, this is near the worst case to operate a direct drive system.

\section{Methodology and Assumptions}

\section{A. Thruster Performance}

Similar to the Refocus Studies ${ }^{3}$, a performance model was created based on provided input of mass flow-rates and thrust as functions of PPU input power. Figure la provides the expected thruster efficiency, however; because the $3 \mathrm{~kW}$ Hall thruster is designed to operate at variable specific impulses, the plasma densities will become too high at the lower $I_{s p}$ to efficiency operate up to $3 \mathrm{~kW}$. The thruster is expected to operate up to approximately $1 \mathrm{~kW}$; the higher voltages allow for a higher maximum power. In order to effectively use the available power, the thrusters in a multi-thruster configuration are designed to operate consecutively as power becomes available. Figure $1 b$ illustrates how the power efficiency of the system can vary at the specified power levels. The throttle table used for mission analysis is therefore derived by theory and not by laboratory data. A larger thruster can be designed for the necessary plasma density, but the mass would increase while the thruster efficiency would likely decrease at the low power operating point.

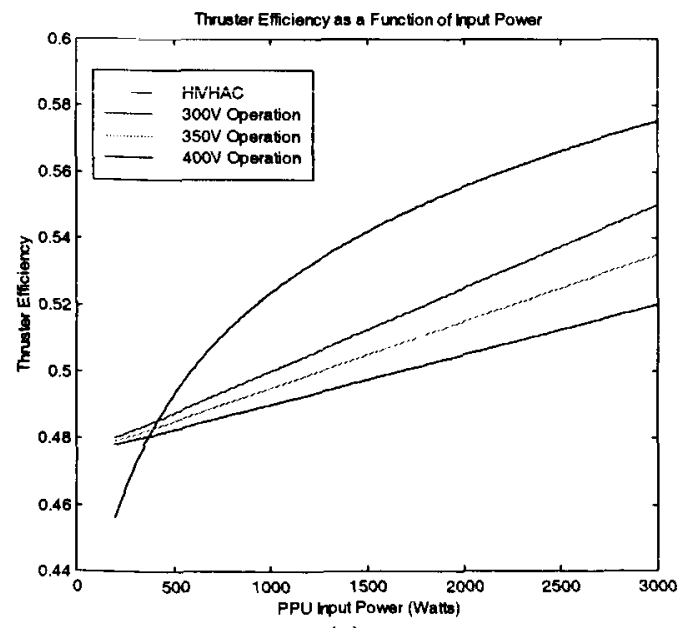

(a)

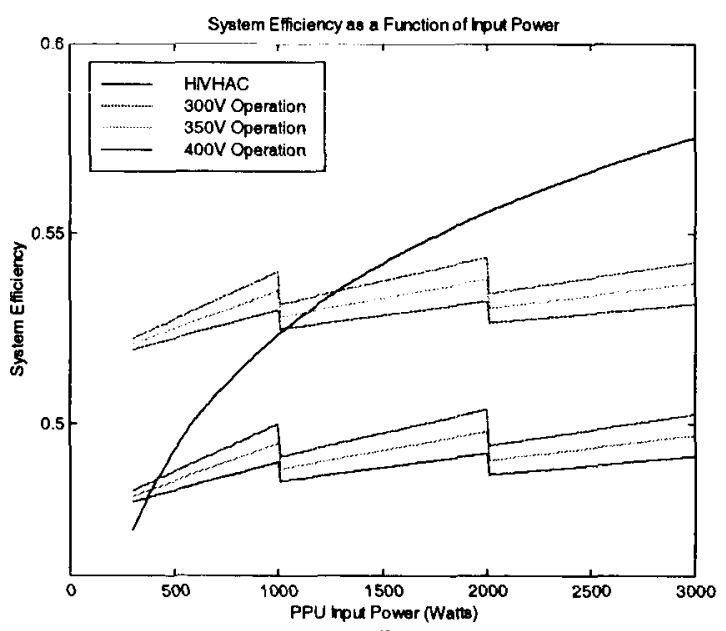

(b) 


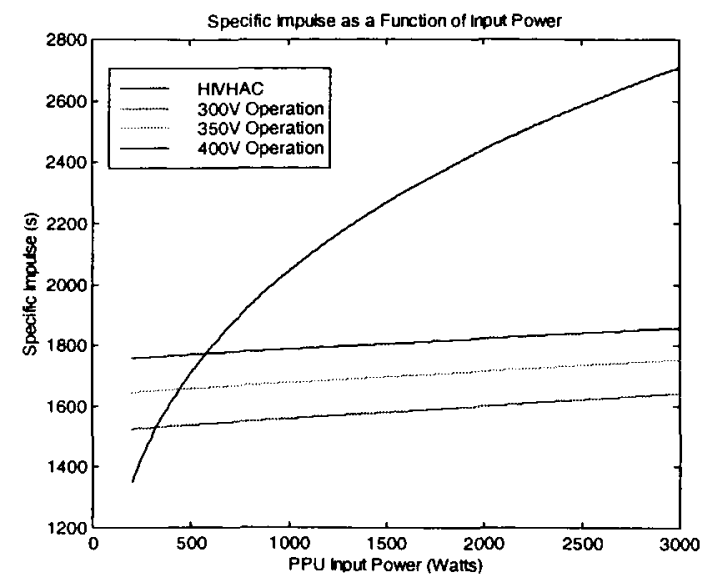

(c)

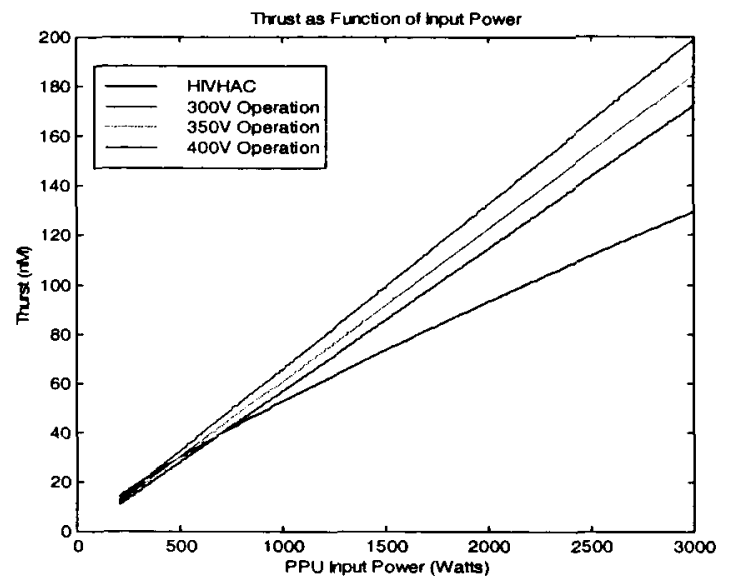

(d)

Figure 1: Theoretical performance of a direct drive Hall thruster compared to the variable specific impulse HiVHAC Thruster.

\section{B. Mass Assumptions}

The subsystem mass breakdown is shown in table 1. The various hardware masses are technology dependent, and the throughput is based on mission analysis. The mass assumptions are consistent with JPL design principles and include mass contingencies based on technology maturity. Of particular interest is the estimated mass of the PPU for the direct drive systems. The savings is due to removing the voltage converter and other mass reductions ${ }^{2}$ associated with direct drive PPUs. Also, it is important to note that only the NSTAR ion thruster has an independent digital control interface unit (DCIU) to provide flow control. These functions are typically incorporated into the PPU for Hall thruster systems. Also, an additional benefit of direct drive systems is the reduced heat transmitted to the spacecraft and a mass penalty is used to account for this savings in this particular study. Table 1 shows that a direct drive system will need more mass than the baseline Hall thruster because of the additional thrusters required. This dry mass increase is first indication that direct drive may not have the desired performance for this particular application. 


\begin{tabular}{|c|c|c|c|c|}
\hline & HIVHAC & $300 V \times 3$ & $400 \mathrm{~V} \times 3$ & NSTAR \\
\hline \multicolumn{5}{|l|}{ Inputs } \\
\hline Number of Engines & 2 & 4 & 4 & 3 \\
\hline Number of PPU's & $\overline{2}$ & 4 & $\overline{4}$ & $\overline{3}$ \\
\hline Number of DCIU's & $\overline{0}$ & 0 & 0 & 2 \\
\hline Xenon Throughput $(\mathrm{kg})^{\star}$ & 238 & 277 & 269 & 194 \\
\hline \multicolumn{5}{|l|}{ Xenon Contingency } \\
\hline Navigation and Trajectory Errors & $5 \%$ & $5 \%$ & $5 \%$ & $5 \%$ \\
\hline Residuals & $5 \%$ & $5 \%$ & $5 \%$ & $5 \%$ \\
\hline \multicolumn{5}{|l|}{ Assumptions } \\
\hline Mass per Thruster & 3.6 & 3.6 & 3.6 & 8.2 \\
\hline Mass per PPU & 8.4 & 4.2 & 4.2 & 13.9 \\
\hline Heat Rejection Hardware ${ }^{\star}$ & 6.1 & 0.6 & 0.6 & 5.2 \\
\hline Mass Per DCIU & 0 & 0 & 0 & 5.7 \\
\hline Mass per Gimbal & 4.6 & 5 & 5 & 4.6 \\
\hline Gimbal Drive Electronics & 2 & 2 & $\overline{2}$ & 0 \\
\hline \multicolumn{5}{|l|}{ Feed System } \\
\hline Fixed Mass & 4 & 4 & $\overline{4}$ & 8.1 \\
\hline Additional Mass/engine & $T$ & 1 & 1 & $\overline{3.3}$ \\
\hline Xenon Tank Mass Fraction & $4.50 \%$ & $4.50 \%$ & $4.50 \%$ & $4.50 \%$ \\
\hline System Contingency & $30 \%$ & $30 \%$ & $30 \%$ & $10 \%$ \\
\hline \multicolumn{5}{|l|}{ Calculations } \\
\hline Thrusters & 7.2 & $\overline{14.4}$ & $\overline{14.4}$ & $\overline{24.6}$ \\
\hline PPUs $^{*}$ & 22.9 & 17.4 & 17.4 & 46.9 \\
\hline DCIUs & 0 & 0 & 0 & 11.4 \\
\hline Feed System & 6 & 8 & 8 & 18 \\
\hline Gimbal & 11.2 & 22 & 22 & 13.8 \\
\hline Subsystem Dry Mass & 47.3 & 61.8 & 61.8 & 114.7 \\
\hline Xenon Residuals & 23.8 & 27.7 & 26.9 & 19.4 \\
\hline Hardware Mass Contingency & 14.19 & 18.54 & 18.54 & 11.47 \\
\hline $\begin{array}{l}\text { Propulsion System } \\
\text { Mass (w/Contingencv) }\end{array}$ & 853 & 108 & $107 ?$ & 1456 \\
\hline Mass Delta (\%) & $-4 !$ & -26 & -26 & Baseline \\
\hline
\end{tabular}

Table 1: Electric Propulsion System Mass Assumptions

\section{Cost Methodology}

The cost of using the electric propulsion system is a primary figure of merit in choosing which system to use on a small cost-capped mission. A detailed cost model is used to estimate the relative cost of each option. The model is based on a combination of flight mission actuals, flight mission estimates, and cost estimates provided by technologists. The cost numbers for this study were performed by JPL with exception of the direct drive PPU costs. The direct drive PPU costs are generated by NAFCOM, a standard cost model based on mass and component complexity. These costs are listed in tables 2 and 3 . Also, non-recurring costs were not included within costs deltas, because the net effect is zero.

The cost estimates fall into four categories including:

1) Development Costs: Cost to develop technology from current state to Technology Readiness Level (TRL) 6. TRL 6 is defined as demonstrated in relevant environment.

2) Qualification Costs: Cost to develop technology from TRL 6 to a fully flight qualified design.

3) Non-recurring Costs: Cost of the post-qualification engineering, data, and drawings for the first flight unit.

4) Recurring Costs: Cost per additional flight units. 


\begin{tabular}{|r|c|c|c|c|}
\hline Cost to Develop and Qual (\$K) & HIVHAC & 300V X 3 & 400V X 3 & NSTAR \\
\hline Thruster Develop & 7000 & 7000 & 7000 & 0 \\
\hline Qual & 2200 & 2200 & 2200 & 0 \\
\hline${ }^{*}$ PPU Develop & 3000 & 845 & 845 & 0 \\
\hline${ }^{*}$ Qual & 1220 & 570 & 570 & 0 \\
\hline DCIU Develop & 0 & 0 & 0 & 0 \\
\hline Qual & 0 & 0 & 0 & 0 \\
\hline Gimbal Develop & 400 & 400 & 400 & 0 \\
\hline Qual & 800 & 800 & 800 & 0 \\
\hline PMS Develop & 2600 & 2600 & 2600 & 0 \\
\hline Qual & 1000 & 1000 & 1000 & 0 \\
\hline SIT & 500 & 500 & 500 & 0 \\
\hline Development Cost & 13500 & 11345 & 11345 & 0 \\
\hline Qual Cost & 5220 & 4570 & 4570 & 0 \\
\hline
\end{tabular}

Table 2: Qualification and Development Cost (\$FY05), Single String

\begin{tabular}{|l|c|c|c|c|}
\hline Component Cost Assumptions (\$K) & HIVHAC & 300V X 3 & 400V X 3 & NSTAR \\
\hline Recurring Cost / Thruster & 250 & 250 & 250 & 580 \\
\hline Recurring Cost / PPU & 940 & 440 & 440 & 1520 \\
\hline Recurring Cost / DClU & 0 & 0 & 0 & 1310 \\
\hline Recurring Cost / Gimbal & 325 & 325 & 325 & 325 \\
\hline Recurring Cost / Xenon Tank & 450 & 450 & 450 & 450 \\
\hline Fixed Cost / Feed System & 1610 & 1610 & 1610 & 1610 \\
\hline Per Thruster Cost/ Feed System & 200 & 200 & 200 & 200 \\
\hline
\end{tabular}

Table 3: Recurring Costs of EPS Hardware.

\begin{tabular}{|l|c|c|c|c|}
\hline Mission Costs (\$K) & HIVHAC & $\mathbf{3 0 0 V ~ X ~ 3}$ & 400V X 3 & NSTAR \\
\hline nth Mission Cost (Comet R) & 5551.4 & 5090.6 & 4989.2 & 12190.6 \\
\hline nth Mission Cost (Nereus) & 4061.8 & 4427.4 & 4417.8 & 9837.8 \\
\hline Delta (Comet R) & -6639.2 & -7100 & -7201.4 & Baseline \\
\hline Delta (Nereus) & -5776 & -5410.4 & -5420 & Baseline \\
\hline First User Cost (Comet R) & 10771.4 & 9660.6 & 9559.2 & 12190.6 \\
\hline First User Cost (Nereus) & 9281.8 & 8997.4 & 8987.8 & 9837.8 \\
\hline Delta (Comet R) & -1419.2 & -2530 & -2631.4 & Baseline \\
\hline Delta (Nereus) & -556 & -840.4 & -850 & Baseline \\
\hline
\end{tabular}

Table 4: First and Nth mission cost of EPS.
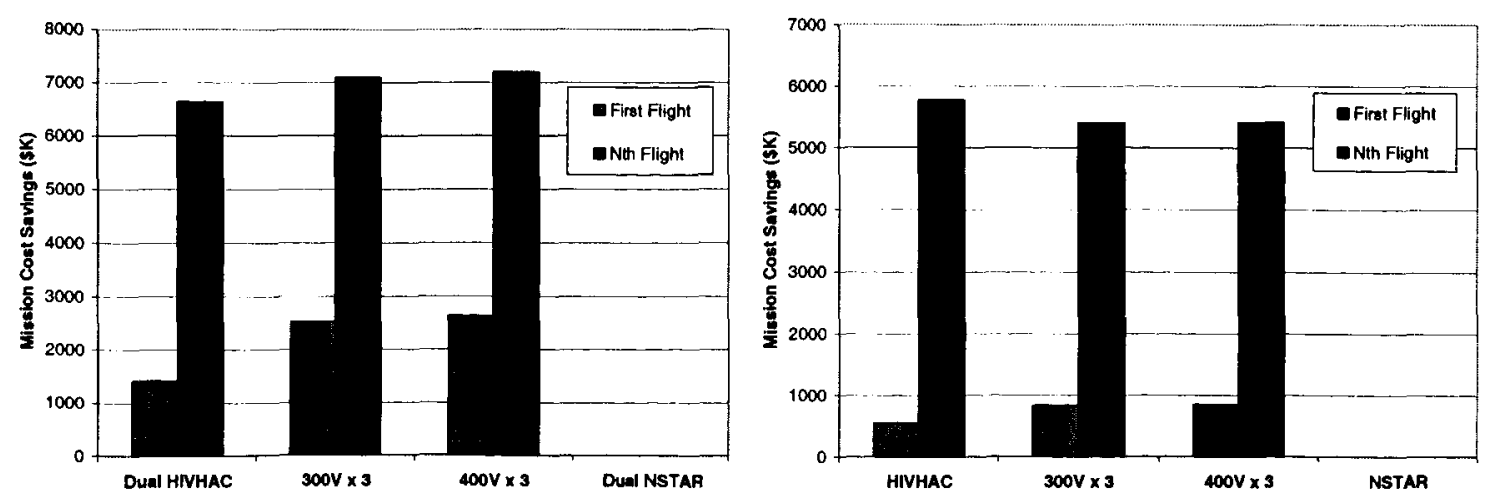

Figure 2: First and Nth mission cost savings of the EPS for Comet Rendezvous (left) and Nereus Asteroid Sample Return (right) missions. 


\section{Systems Analysis Results}

Mission analysis and trajectory calculations for this study were performed using SEPTOP. Typically, trajectory analysis will be run to optimize the $I_{s p}$ for a given power and destination. Because the voltages are held constant, the $I_{s p}$ is held constant, therefore the throughput and thrust varies proportionally with input power. As previously mentioned, he HiVHAC thruster designed to operate at $800 \mathrm{~V}$ and $3 \mathrm{~kW}$ cannot operate at $3 \mathrm{~kW}$ at $300 \mathrm{~V}$ because the plasma density would increase beyond the thrusters design. Therefore rather than scaling a larger thruster that is inefficient at very low powers, additional thrusters can be used to provide the desired thrust. The addition of thrusters will increase the system mass and complexity, but there is test heritage of multiple Hall thrusters.

The two missions selected for study are the Nereus Asteroid Sample Return and the Kopff Comet Rendezvous mission. The missions were selected to be consistent with the re-focus studies which provide a baseline for comparison. The Nereus mission involves launching with an Earth escape trajectory, using solar electric propulsion to rendezvous with the asteroid, remain at the asteroid for 90 days, and then conduct an Earth flyby for sample release. The Kopff comet rendezvous mission is also designed to launch with an Earth escape trajectory and use SEP to rendezvous with the comet.

\section{A. Nereus Asteroid Sample Return}
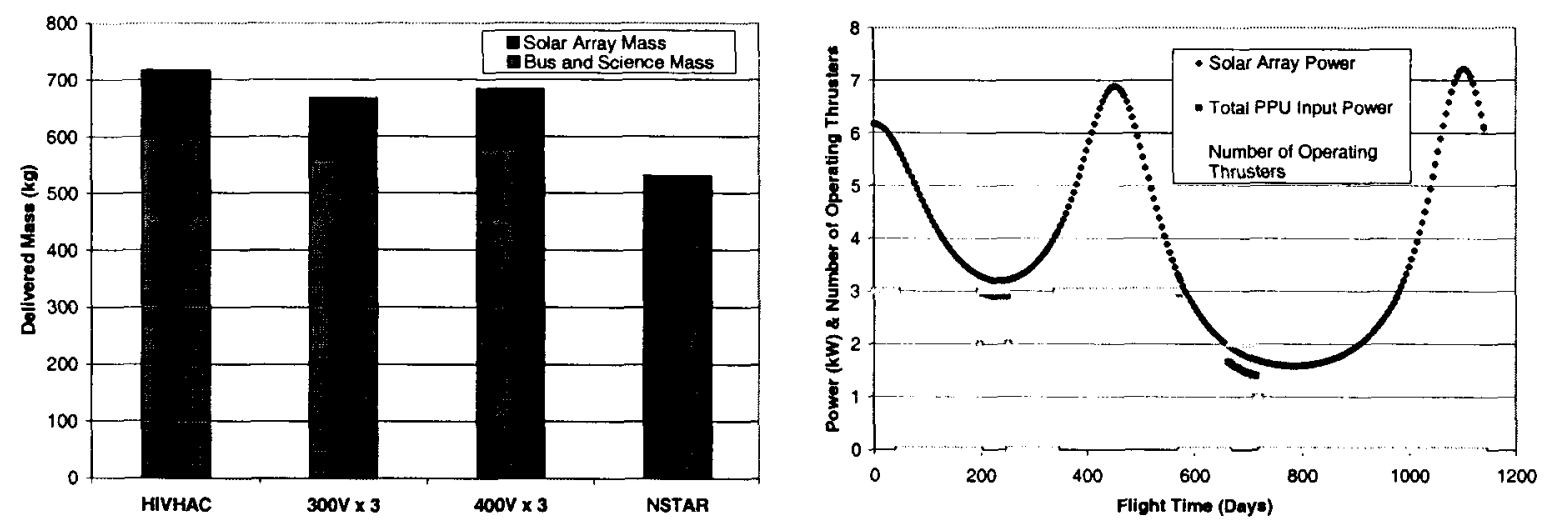

Figure 2: Delivered mass (left) and mission profile (right) for Nereus sample return mission.

\begin{tabular}{|l|c|c|c|c|}
\hline Nereus Asteroid Sample Return & HIVHAC & 300V X 3 & 400V X 3 & NSTAR \\
\hline Launch Vehicle & Delta II 2925 & Delta II 2925 & Delta II 2925 & Delta II 2925 \\
\hline C3 (km2/s2) & 4.3 & 3.77 & 3.52 & 11.8 \\
\hline Trip Time (yrs) & 3.15 & 3.11 & 3.12 & 3.25 \\
\hline Power (1 AU, BOL) kW & 6 & 6 & 6 & 6 \\
\hline Net Payload Mass Delivered & 717 & 669 & 685 & 531 \\
\hline Launch Mass (kg) & 1181 & 1194 & 1201 & 1010 \\
\hline Xenon Propellant Throughput & 239 & 277 & 269 & 194 \\
\hline Net Trajectory Mass Capability & 802 & 777 & 792 & 677 \\
\hline Propulsion Subsystem Mass & 85.3 & 108 & 107.2 & 145.6 \\
\hline Mission Delta V (km/s) & 5.86 & 4.1 & 4.52 & 6.5 \\
\hline Throughput required per thruster (kg) & 238 & 92 & 90 & 194 \\
\hline
\end{tabular}

Table 5: Summary of results of Nereus mission analysis. 


\begin{tabular}{|l|c|c|c|c|}
\hline Nereus Asteroid Sample Return & $400 \mathrm{~V}$ X 3 & $400 \mathrm{~V}$ X 4 & 400V X 5 & 400V X 6 \\
\hline Launch Vehicle & Delta II 2925 & Delta II 2925 & Delta II 2925 & Delta II 2925 \\
\hline C3 (km2/s2) & 3.52 & 3.02 & 2.76 & 2.63 \\
\hline Trip Time (yrs) & 3.12 & 3.11 & 3.11 & 3.1 \\
\hline Power (1 AU, BOL) kW & 6 & 6 & 6 & 6 \\
\hline Net Payload Mass Delivered & 685 & 698 & 693 & 681 \\
\hline Launch Mass (kg) & 1201 & 1213 & 1220 & 1223 \\
\hline Xenon Propellant Throughput & 269 & 252 & 246 & 243 \\
\hline Net Trajectory Mass Capability & 792 & 821 & 834 & 840 \\
\hline Propulsion Subsystem Mass & 107.2 & 123.5 & 140.8 & 158.8 \\
\hline Mission Delta V (km/s) & 4.52 & 4.15 & 4.01 & 3.94 \\
\hline Throughput required per thruster (kg) & 90 & 63 & 49.2 & 40.5 \\
\hline
\end{tabular}

Table 6: Comparison of additional thrusters for Nereus mission analysis.

\section{B. Kopff Comet Rendezvous}
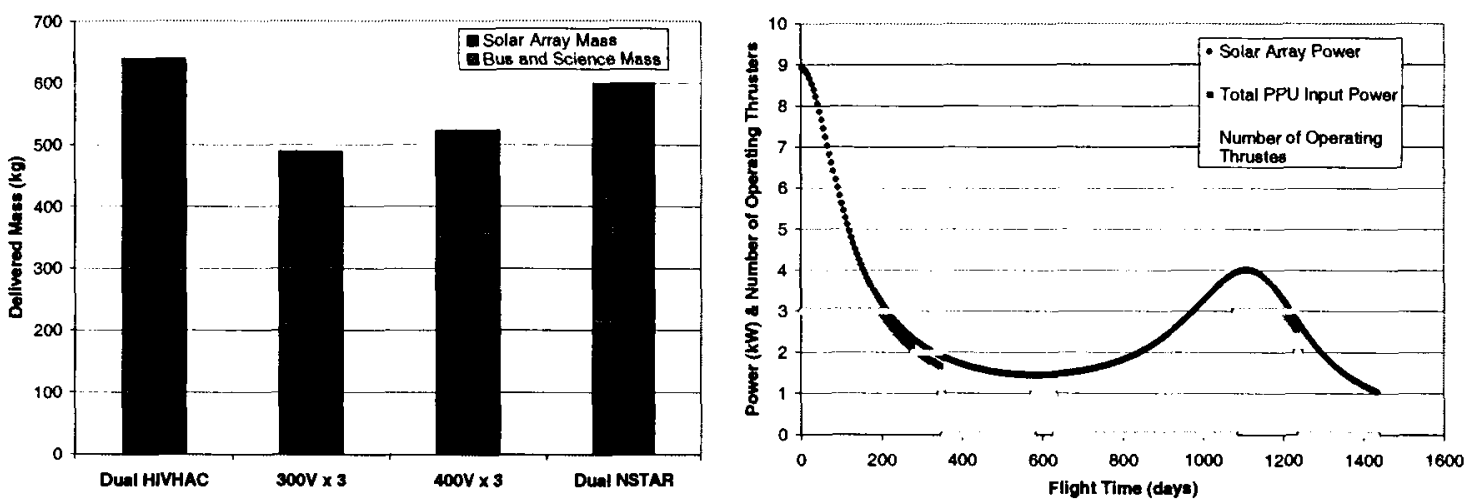

Figure 3: Delivered mass (left) and mission profile (right) for Kopff comet rendezvous mission.

\begin{tabular}{|l|c|c|c|c|}
\hline Comet Rendezvous & Dual HIVHAC & 300V X 3 & 400V X 3 & Dual NSTAR \\
\hline Launch Vehicle & Delta II 2925H & Delta II 2925H & Delta II 2925H & Delta II 2925H \\
\hline C3 (km2/s2) & 11.49 & 10.3 & 12.93 & 15.8 \\
\hline Trip Time (yrs) & 3.85 & 3.93 & 3.89 & 3.83 \\
\hline Power (1 AU, BOL) kW & 9 & 9 & 9 & 9 \\
\hline Net Payload Mass Delivered & 639 & 489 & 523 & 599 \\
\hline Launch Mass (kg) & 1079 & 1106 & 1047 & 987 \\
\hline Xenon Propellant Throughput & 322 & 488 & 403.5 & 238 \\
\hline Net Trajectory Mass Capability & 756.47 & 618 & 643.64 & 749 \\
\hline Propulsion Subsystem Mass & 117 & 129 & 121 & 150 \\
\hline Mission Delta V (km/s) & 8.77 & 8.87 & 8.54 & 8.5 \\
\hline Throughput required per thruste & 161 & 163 & 134.5 & 119 \\
\hline
\end{tabular}

Table 7: Summary of results of Kopff mission analysis.

Based on these two cases, some potential exists for direct drive even at low power operation. The Nereus missions shows that direct drive system does have performance benefit and significant cost benefit over the SOA NSTAR thruster, however; the performance is not as good as the variable specific impulse Hall thruster. The direct drive system does still have cost benefit because of the reduction in solar arrays, even with the cost of additional EPS hardware. In fact, the smaller solar array will also save an additional $10.8 \mathrm{~kg}$ therefore the performance difference between the HiVHAC and the direct drive system is only about 5\%. From the perspective of hardware costs, the cost of additional thrusters outweighs the cost savings of a direct drive PPU, instead; it is the secondary cost savings of the solar array that makes direct drive more economical. 
The comet rendezvous mission is not very good for a direct drive system limited to 400 volts. This is because the $\Delta V$ is very large and the $I_{s p}$ limit is detrimental. Attempting the comet rendezvous mission with four or five thrusters does not give any additional payload benefit because the propellant throughput grows to over $500 \mathrm{~kg}$. This shows for large $\Delta \mathrm{V}$ missions, the exponential effect of the rocket equation and specific impulse outweighs the dry mass advantage of a direct drive system.

\section{Remarks on Application}

\section{A. Deep Space versus Near Earth Missions}

The majority of negative characteristics found in using a low power direct drive system are only present in science missions involving a varying input power. It is the deep throttle effect that reduces the benefits of direct drive. Very low power operation designed to run at multiple specific impulses is a worst case scenario for a direct drive system.

For a direct drive system that will function in a geostationary, or any other obit that will receive relatively constant power conditions, the gains are evident without the difficulties in performance optimization and mission planning. A spacecraft that will receive a near constant power throughout the life of its mission can have a direct drive thruster optimized for the one condition at which it will operate. The limiting factor of direct drive for LEO/GEO type spacecraft is typically the higher plasma densities. A near Earth spacecraft operating at very high, several hundred, voltages may experience arcing. Progress has been made in solar array technology to prevent arcing up to approximately $400 \mathrm{~V}$; more than enough for direct drive Hall thrusters. Spacecraft that are operating near the Earth also do not need to compensate for low power, High AU, thrusting because there is no power fall-off as in deep-space missions. For example, a lunar or Mars cargo tug will complete its entire mission with very little change in available input power and should therefore benefit from the large initial dry mass savings. Also, as other studies have shown, the higher the available power, the more significant the direct drive savings become.

\section{B. Dependence on Power, Specific Impulse, and $\Delta V$}

It has been noted in numerous studies that direct drive is a method of reducing system mass, either for the delivery of more payload or for additional propellant to increase the operational period of orbit maintenance. By simply using the known specific masses of the subsystems and solar arrays in conjunction with the rocket equation, one can trade the benefits of direct drive application versus power.

As shown in figure 4, for a system designed to be run as either direct drive or with an adjustable specific impulse, the missions that optimize to an $I_{\mathrm{sp}}$ higher than achievable with direct drive tend to show direct drive as a poor performer, especially at low power. Also, for missions that require large $\Delta V$ 's, the inability to achieve the desired $I_{\mathrm{sp}}$ becomes even more significant.

As the power levels increase, the cost and mass savings of a direct system become very significant. Th use of direct drive becomes a trade of prope!lant mass with system mass. A high $I_{s p}$ thruster will consume less propellant, however; lower system masses can reduce the inert fraction of the rocket equation.

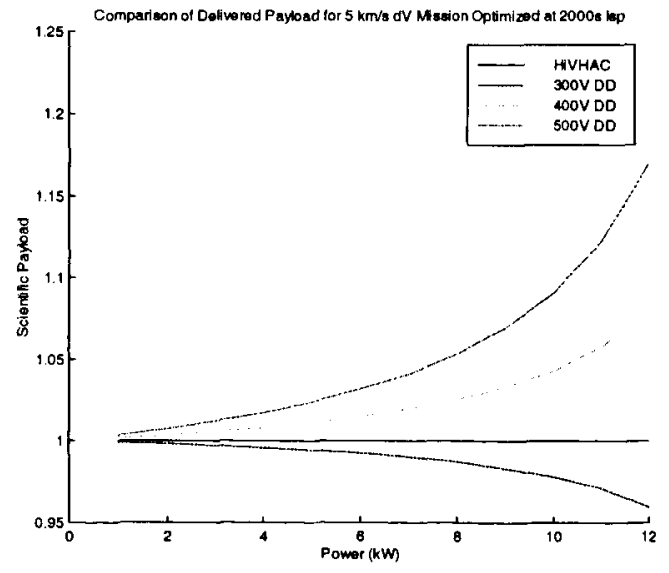

(a)

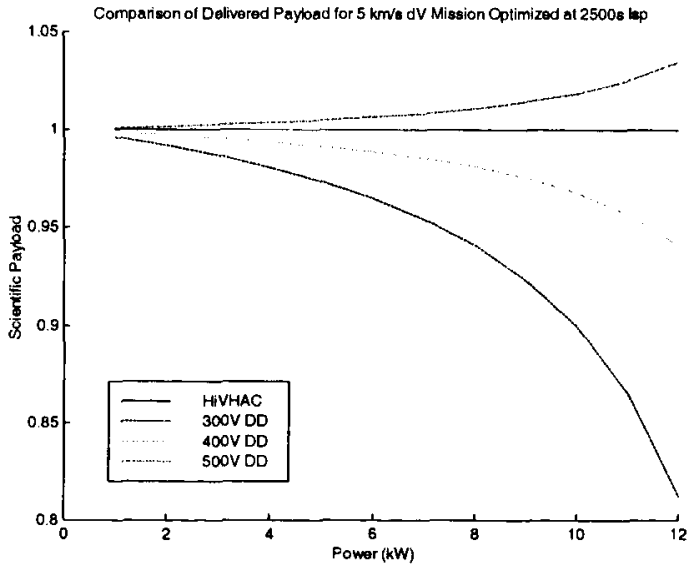

(b) 


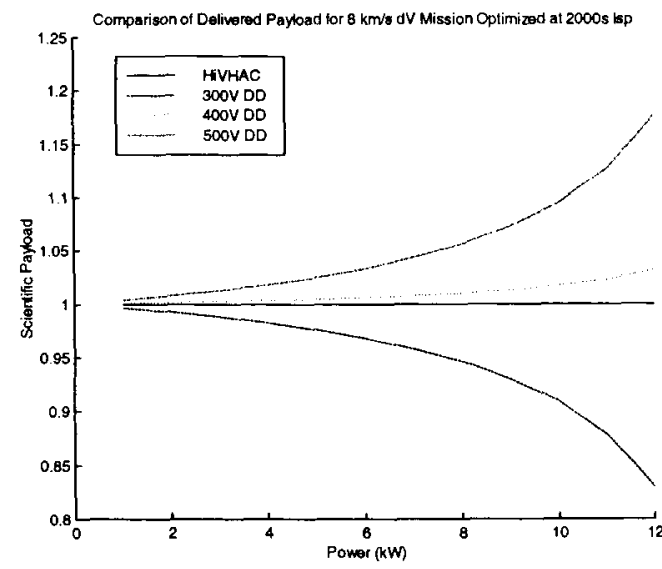

(a)

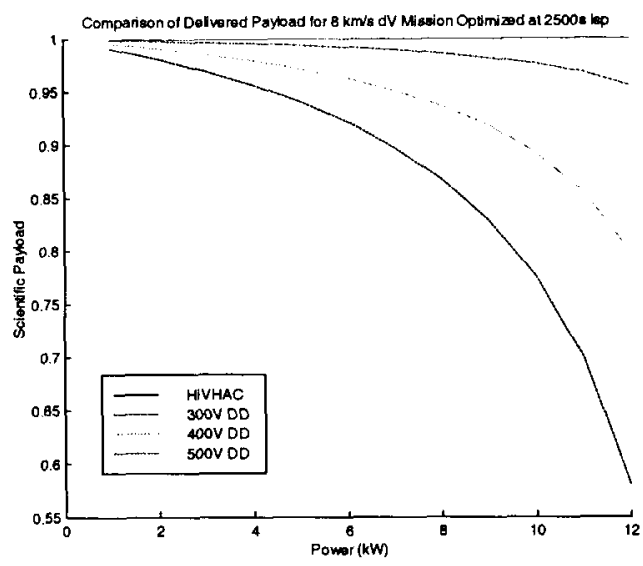

(b)

Figure 4: Normalized payload to final destination for multiple specific impulse and $\Delta V$ requirements.

\section{System Configuration}

Clusters of thrusters have been demonstrated for Hall thrusters with individual or shared cathodes without any change in performance. One cathode can be configure to operate at low current levels for 1-2 thrusters, and a second larger cathode can be optimized for 3-4 thruster operation. The dual cathode design should allow for the system to operate without additional keeper power when available power is at a premium. It should be noted that one benefit of direct drive not accounted for in this study, is the ability of the PPU to operate above $90 \%$ efficiency at all conditions. It will be a non-trivial task to develop a PPU for the low power Hall thruster than can also operate efficiently at a wide range of power conditions. Typically a PPU is designed for peak performance at peak power and may only show efficiencies in the high eighties near its low power limitations.

\section{Conclusions}

The direct drive Hall thruster system is capable of higher performance and significantly lower costs than the $\mathrm{SOA}$ ion propulsion systems. Large throttling or high $\mathrm{I}_{\mathrm{sp}}$ missions diminish the benefits of direct drive compared to the HiVHAC thruster designed to operate at variable specific impulses. The performance of the HiVHAC thruster is higher than achievable with the thruster running at a constant voltage level; however the increased system efficiency by removing the PPU can compensate for some of the performance losses. The cost of a smaller solar array is a significant savings of direct drive application. For large $\Delta \mathrm{V}$ missions, the exponential effect of specific impulse on the rocket equation becomes more significant than dry mass reduction.

The use of a direct drive system can be another method of reaching the goals of the In-Space program; including a low complexity, low cost electric propulsion system. Also, the need of running additional thrusters does have the additional benefit of reducing the required propellant throughput per thruster. Because the benefits of direct drive increase with higher power systems, as well as missions with a smaller throttle range, this is a worst case scenario for a direct drive application. With the knowledge that this worst case direct drive system can demonstrate benefits, it should be emphasized that the gains are too large to be ignored on larger systems.

\section{Future Work}

A direct drive low power Hall thruster may not produce the significant benefits that can be seen with larger, higher powered systems. However, missions moving towards the sun, such as inner planetary missions; may not exhibit any loss in performance due to rising solar array voltages. Inner planetary missions in general may benefit from low power Hall systems because any redundancy, such as multiple thrusters, such as unused EP strings at the beginning of the mission will be less cumbersome due to the reduced mass and complexity compared to that of a PPU controlled Hall thruster system. The ability to thrust at the low-power throttle range, in this case near the Earth, may not play as significant role in the mission analysis and should be traded. Also, tug missions which perform large delta V's near a gravity field may optimize closer to the lower and constant direct drive $\mathrm{I}_{\mathrm{sp}}$ because the higher thrust levels may reduce the gravity losses. As mentioned, a larger thruster can be used to compensate for higher plasma densities at high power if the exhaust velocity is limited, and this trade should also be characterized. 


\section{Acknowledgments}

The work described in this paper was funded in whole or in part by the In-Space Propulsion Technology Program, which is managed by NASA's Science Mission Directorate in Washington, D.C., and implemented by the In-Space Propulsion Technology Office at Marshall Space Flight Center in Huntsville, Ala. The program objective is to develop in-space propulsion technologies that can enable or benefit near and mid-term NASA space science missions by significantly reducing cost, mass or travel times. The author would like to especially thank David Manzella (GRC) for thruster performance data and numerous communications on direct drive requirements, and Tara Polsgrove (MSFC) for her help in the low thrust mission analysis.

\section{References}

${ }^{1}$ Hamley, John A., 1995 "Direct Drive Options for Electric Propulsion Systems.” NASA TM 106576

${ }^{2}$ Hoskins, et.al., "Direct Drive Hall Thruster Systems Development," AIAA-03-4726, $39^{\text {th }}$ AIAA/ASME/SAE/ASEE Joint Propulsion Conference, Huntsville, AL, July 20-23, 2003

${ }^{3}$ Oh, David, "Evaluation of Solar Electric Propulsion Technologies for Discovery Class Missions," AIAA-2005-4270, $41^{\text {st }}$ Joint Propulsion Conference, Tucson, AZ, July 10-13, 2005.

${ }^{4}$ Jet Propulsion Laboratory, California Institute of Technology, "Solar Cell Array Design Handbook," NASA JPL SP 43-38, October 1976

${ }^{5}$ Witzberger, K., Benson, S., Manzella, D., Oh, D., Brophy, J., and Cupples, M., "NASA's 2004 In-Space Propulsion ReFocus Studies for New Frontiers Class Missions," AIAA-2005-4271, $41^{\text {st }}$ Joint Propulsion Conference, Tucson, AZ, July 10-13, 2005. 